\title{
A simple and inexpensive telemetry system for measuring deep body temperature in rodents
}

\author{
WILLIAM C. GORDON, KERRY L. COBURN, and ZITA M. WENZEL \\ State University of New York, Binghamton, New York 13901
}

\begin{abstract}
An easily constructed telemetry system for measuring deep body temperatures in rodents is described and data collected with the system is presented. The system allows continual monitoring of core temperature without interfering with concomitant behavioral or physiological measurements.
\end{abstract}

Growing interest in the relationship between circadian rhythms and a variety of behavioral measures (cf. McFarland, 1975; Stroebel, 1967) has arisen partly because measures of learning, retention, and motivation have been shown to vary considerably as a function of the time of day these measures are taken (e.g., Stroebel, 1967; Ternes, 1976). Likewise, a number of the treatments used to study learning, memory, or motivational processes appear to be differentially effective depending on the time of day the treatments are administered (e.g., Scheving, Vedral, \& Pavly, 1968; Stephens \& McGaugh, 1968; Gordon, Wenzel, Coburn, \& Levine, Note 1).

One of the most reproducible and most often studied biological processes exhibiting circadian periods is deep body temperature. Usually the deep body temperature of rodents is measured by rectal probes, which either are inserted at the time of measurement or are inserted and then taped to the tail of the animal to mimic an indwelling temperature probe (e.g., Meeter \& Harting, 1975; Stephens \& McGaugh, 1968). While these techniques result in stable measures of deep body temperature, the use of either type probe presents problems whenever concomitant measures of several cyclic functions are taken. For example, the hourly handling of animals to insert a probe can alter an animal's activity levels. It is equally possible that eating and drinking cycles, corticosterone levels in the blood, and urination or defecation rates may be altered by the handling procedures necessary with this technique.

When the temperature probe is inserted and fastened to an animal's tail, a similar set of problems arises. There is interference with normal defecation rates even if the probe is small, and the technique may also interfere with normal urination and activity, or induce changes in corticosterone levels.

Preparation of this manuscript was supported by a grant from the Biomedical Research Support Grants Program, State University of New York at Binghamton, awarded to the first author. Requests for reprints should be addressed to William C. Gordon, Department of Psychology, State University of New York at Binghamton, Binghamton, New York 13901.
One solution is to implant a thermistor in a location other than the rectum, and to monitor the unit with some type of telemetry system. In most cases the cost of such a system for even one animal is prohibitive.

The temperature telemetry system described here is relatively inexpensive, does not interfere with activity, defecation, or urination rates, and provides stable measures of deep body temperature.

\section{THE TELEMETRY UNIT AND IMPLANTATION}

The telemetry unit employed (Model V, Mini-Mitter Corporation, Indianapolis, Indiana; approximate cost, $\$ 20$ ) contains a temperature-sensitive resistive element as a thermistor, which emits an rf signal modulated by an auditory signal (a "click" sound). The thermistor is encased in a nontoxic plastic cylinder, $19 \mathrm{~mm}$ long and $12 \mathrm{~mm}$ in diam. The click sound, which can be picked up on an AM broadcast-band radio, increases in frequency as the surrounding temperature increases. The precise relationship between degrees centigrade and the number of clicks emitted per unit time is obtained by calibrating the telemetry unit in a heated bath prior to implantation. The entire telemetry unit weighs $2.3 \mathrm{~g}$ and is capable of transmitting a signal approximately $3 \mathrm{ft}$.

While the plastic capsule surrounding the thermistor is moisture resistant, it is helpful to seal the capsule to retard corrosion when the unit is to remain in an animal for long periods. Wrapping the capsule in a piece of surgical glove usually suffices. The wrapped capsule is implanted in the I.P. cavity of the rat between the sniall intestine and the abdominal wall. Since surgery involves only a small incision in the abdominal wall, animals are usually fully recovered within a week.

\section{TELEMETRY RECORDING SYSTEM}

Clear reception of the auditory signal is obtained by placing an implanted animal in a standard vivarium cage $(20 \times 24 \times 18 \mathrm{~cm})$ and mounting a small transistor radio inside the cage. Signals are then transmitted from several 
radios, each at specified times, to counters or recording devices via a simple electromechanical circuit. The transmission and recording of the auditory signals can be accomplished in a variety of ways.
Figure 1 presents a circuit diagram for using a tape recorder to record the signals for later transcription. The output from each radio phone jack is connected to the input jack of a tape recorder via separate DPDT

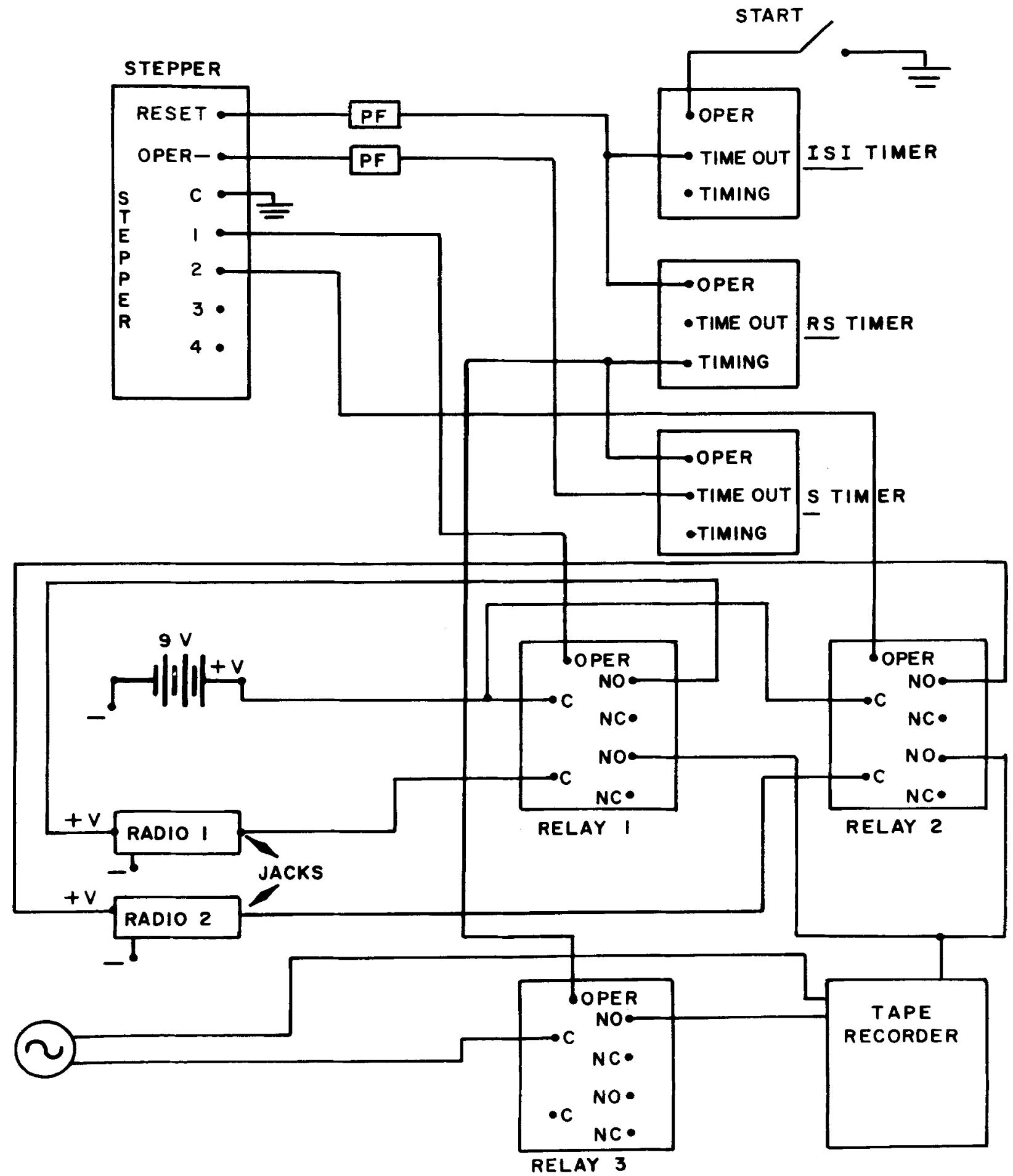

Figure 1. Circuit diagram for temperature telemetry system. 
relays. To record the signals from several radios in a given period, it is necessary to operate the multiple DPDT relays (each corresponding to a separate radio) through different positions of a stepper, which in turn is controlled by a timing circuit. The timing circuit consists of three recycling timers. The intersession interval (ISI) timer controls the time between recording sessions; the recording session (RS) timer controls the length of a recording session; and the sample (S) timer controls the length of time each radio is monitored within a given recording session. Since each position of the stepper operates a different relay representing a different radio, this system allows the sequential recording of several radio signals, each for a specified period of time.

In this circuit, power (ac) is supplied to the tape recorder only during the RS period. This is accomplished by routing the common wire of the power cord through a relay operated by the onset of the RS timer. The relay must be isolated from the rest of the circuit to reduce shock hazard.

\section{EXPERIMENTAL APPLICATION}

In measuring circadian fluctuations in deep body temperature, it is useful to record temperatures hourly. This entails using an intersession interval of $1 \mathrm{~h}$ minus the duration of the recording session. The duration of the recording session depends on the period of time each animal is monitored and the number of animals monitored. Usually a sampling period of $1 \mathrm{~min}$ per recording session is sufficient for each animal. This means that the $\mathrm{S}$-timer duration should be set at $1 \mathrm{~min}$. However, it is useful to program in at least $1 \mathrm{~min}$ of blank recording at the end of each recording session, so that recording sessions can be discriminated easily when the tapes are transcribed.

Using this system, we have been able to obtain daily temperature rhythms that closely approximate those reported by investigations using rectal temperature probes (cf. Halberg, 1960; Stephens \& McGaugh, 1968). Figure 2 illustrates representative temperature cycles obtained with this system for animals housed with $12 \mathrm{~h}$ of light $(0900-2100 \mathrm{~h})$ and $12 \mathrm{~h}$ of darkness (2100$0900 \mathrm{~h}$ ). These cycles reflect the mean temperature measures of four male albino rats taken on 4 consecutive days. Temperature cycles for each animal are similar in amplitude and phase to the group curves. Also, measures for individual animals tend to be very consistent across

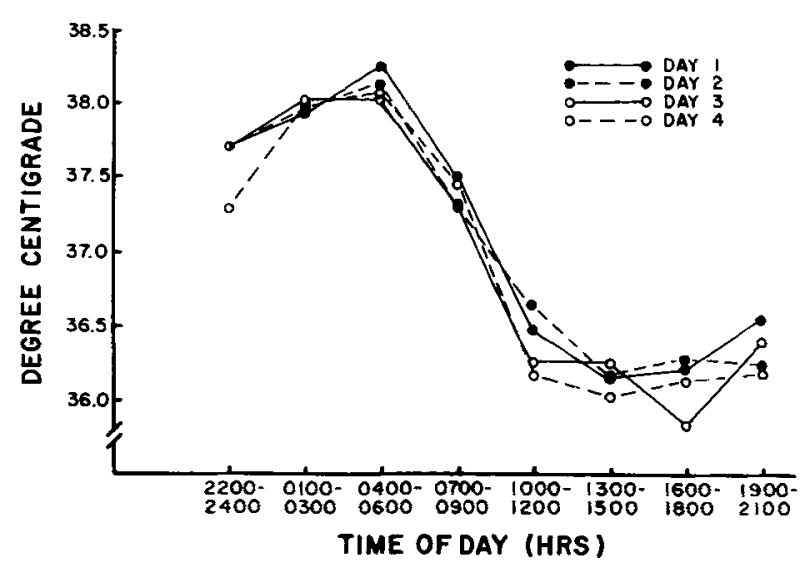

Figure 2. Mean temperature for four rats on four consecutive days as a function of time of day.

consecutive days of recording. In most cases the correlation (Pearson $r$ ) between an animal's temperature measures on consecutive days is between +.75 and +1.00 .

\section{REFERENCE NOTE}

1. Gordon, W. C., Wenzel, Z. M., Coburn, K. L., \& Levine, J. Effects of ECS on circadian fluctuations in deep body temperature and gross motor activity. Unpublished manuscript, 1977. (Available from Department of Psychology, State University of New York at Binghamton. Binghamton, New York.)

\section{REFERENCES}

Halberg, F. Temporal coordination of physiological function. In Cold Harbor Symposia on Quantitative Biology, 1960, 25, 289-310.

MCFARLAND, R. A. Air travel across time zones. American Scientist, 1975, 63, 23-30.

Meeter, E., \& Hartung, J. W. The effect of pentylenetetrazol on the body temperature of the rat. In P. Lomax, E. Schonbaum, \& J. Jacobs (Eds.), Temperature regulation and drug action. New York: Karger, 1975. Pp. 284-292.

Scheving, L. E., Vedral, D. F., \& Pavly, J. E. Daily circadian rhythm in rats to d-amphetamine sulphate: Effect of blinding and continuous illumination on the rhythm. Nature, 1968. 219. 621-622.

Stephens, G., \& McGaugh, J. L. Retrograde amnesia: Effects of periodicity and degree of training. Communications in Behavioral Biology, 1968, 267-275.

Stroebel, C. F. Behavioral aspects of circadian rhythms. In J. Zubin \& H. F. Hunt (Eds.), Comparative psychopathology: Animal and human. New York: Grune \& Stratton, 1967. Pp. 158-171.

Ternes, J. W. Resistance to extinction of a learned taste aversion varies with time of conditioning. Animal Learning \& Behavior. 1976. 4, 317-321.

(Received for publication November 29, 1976; revision accepted February 16, 1977.) 